\title{
Insights on early mutational events in SARS-CoV-2 virus reveal founder effects across geographical regions
}

\author{
Carlos Farkas ${ }^{\text {Corresp., } 1,2}$, Francisco Fuentes-Villalobos ${ }^{3}$, Jose Luis Garrido ${ }^{4}$, Jody Haigh ${ }^{1,2}$, María Inés Barría ${ }^{\text {Corresp. } 3}$ \\ 1 Oncology and Hematology, CancerCare Manitoba, Winnipeg, Manitoba, Canada \\ 2 Department of Pharmacology and Therapeutics, Rady Faculty of Health Sciences, University of Manitoba, Winnipeg, Manitoba, Canada \\ 3 Faculty of Biological Sciences, Department of Microbiology, Center of Biotechnology, Universidad de Concepción, Universidad de Concepción, \\ Concepción, Chile \\ 4 Ichor Biologics LLC, New York, New York, United States \\ Corresponding Authors: Carlos Farkas, María Inés Barría \\ Email address: carlos.farkaspool@umanitoba.ca, mbarriac@udec.cl
}

Here we aim to describe early mutational events across samples from publicly available SARS-CoV-2 sequences from the sequence read archive and GenBank repositories. Up until March 27, 2020, we downloaded 50 illumina datasets, mostly from China, USA (Washington State) and Australia (Victoria). 30 datasets (60\%) contain at least a single founder mutation and most of the variants are missense (over 63\%). Five-point mutations with clonal (founder) effect were found in USA next-generation sequencing samples. Sequencing samples from North America in GenBank (April 22, 2020) present this signature with up to $39 \%$ allele frequencies among samples $(n=1359)$. Australian variant signatures were more diverse than USA samples, but still, clonal events were found in these samples. Mutations in the helicase, encoded by the ORFlab gene in SARS-CoV-2 were predominant, among others, suggesting that these regions are actively evolving. Finally, we firmly urge that primer sets for diagnosis be carefully designed, since rapidly occurring variants would affect the performance of the reverse transcribed quantitative PCR (RT-qPCR) based viral testing. 


\section{Insights on early mutational events in SARS-CoV-2}

2 virus reveal founder effects across geographical

3 regions

4

5

6

7

8

Authors: Carlos Farkas ${ }^{1,2 *}$, Francisco Fuentes-Villalobos ${ }^{3}$, José Luis Garrido ${ }^{4}$, Jody J, Haigh ${ }^{1,2}$ and María Inés Barría ${ }^{3 *}$

${ }^{1}$ Research Institute in Oncology and Hematology, CancerCare Manitoba, Winnipeg, Manitoba, Canada.

${ }^{2}$ Department of Pharmacology and Therapeutics, Rady Faculty of Health Sciences, University of Manitoba, Winnipeg, Manitoba, Canada

${ }^{3}$ Faculty of Biological Sciences, Department of Microbiology, Center of Biotechnology, Universidad de Concepción, 4070386 Concepción, Chile.

${ }^{4}$ Ichor Biologics LLC, New York, NY 10065, USA.

Corresponding Author(s):

Carlos Farkas ${ }^{1,2}$

Research Institute in Oncology and Hematology, CancerCare Manitoba, ON5029, 675 McDermot Ave, Winnipeg, MB, Canada, R3E 0V9

Email address: carlos.farkaspool@umanitoba.ca

María Inés Barría ${ }^{3}$

${ }^{3}$ Faculty of Biological Sciences, Department of Microbiology, Center of Biotechnology, Universidad de Concepción, 4070386 Concepción, Chile Email address: mbarriac@udec.cl

\section{Abstract}

Here we aim to describe early mutational events across samples from publicly available SARSCoV-2 sequences from the sequence read archive and GenBank repositories. Up until March 27, 2020, we downloaded 50 illumina datasets, mostly from China, USA (Washington State) and Australia (Victoria). 30 datasets (60\%) contain at least a single founder mutation and most of the variants are missense (over 63\%). Five-point mutations with clonal (founder) effect were found in USA next-generation sequencing samples. Sequencing samples from North America in GenBank (April 22, 2020) present this signature with up to $39 \%$ allele frequencies among samples $(n=1359)$. Australian variant signatures were more diverse than USA samples, but still, clonal events were found in these samples. Mutations in the helicase, encoded by the ORF1ab gene in SARS-CoV-2 were predominant, among others, suggesting that these regions are actively evolving. Finally, we firmly urge that primer sets for diagnosis be carefully designed, since rapidly occurring variants would affect the performance of the reverse transcribed quantitative PCR (RT-qPCR) based viral testing. 
40

41

42

43

44

45

46

47

48

49

50

51

52

53

54

55

56

57

58

59

60

61

62

63

64

65

66

67

68

69

70

71

72

73

74

75

76

77

78

79

\section{Introduction}

The COVID-19 pandemic caused by a novel 2019 SARS coronavirus, known as SARS-CoV-2, is rapidly spreading worldwide, greatly surpassing the 8000 total cases of the 2002-2004 SARS coronavirus outbreak (SARS-CoV-1) after one month of the initially identified case on December 31, 2019, in Wuhan city, China (Wilder-Smith et al. 2020). As SARS-CoV-2 is human-to-human transmitted, it is a threat to the global population. It is critical to understand SARS-CoV-2 characteristics to deal with this ongoing pandemic and to develop future treatments. SARS-CoV2 virus is an enveloped, positive-stranded RNA virus with a large genome $(29.9 \mathrm{~kb})$ belonging to the family Coronaviridae, order Nidovirales (de Wit et al. 2016). One of the striking genomic features of this novel virus is the presence of a novel furin-like cleavage site in the S-protein of the virus, which differs from SARS-CoV-1 and may have implications for the life cycle and pathogenicity of the novel virus (Coutard et al. 2020; Wu et al. 2020a). Firstly, it was suggested that SARS-CoV-2 is a close relative of the RaTG13 bat-derived coronavirus (around 88\% identity) rather than of SARS-CoV-1 (79\% identity) or middle east respiratory syndrome coronavirus MERS-CoV (50\% identity) (Lu et al. 2020). Due to this association with bat coronaviruses, it was also argued that SARS-CoV-2 virus has the potential to spread into another species, as bat coronaviruses do (Hu et al. 2018). Although bats are likely natural reservoir hosts for SARS-CoV2 , recently it was demonstrated that SARS-CoV-2 is closely related to a pangolin coronavirus (Pangolin-CoV) found in dead Malayan pangolins with a $91.02 \%$ identity, the closest relationship found so far for SARS-CoV-2 (Zhang et al. 2020). In that study, genomic analyses revealed that the $\mathrm{S} 1$ protein of Pangolin-CoV is related closer to SARS-CoV-2 than to RaTG13 coronavirus. Also, five key amino acid residues involved in the interaction with the human ACE2 receptor are maintained in Pangolin-CoV and SARS-CoV-2, but not in RaTG13 coronavirus. Thus, it is likely pangolins are an intermediate host in the transmission of coronaviruses between bats and humans. In this manner, it was argued SARS-CoV-2 acquired mutations needed for human transmission and will continue to evolve with novel mutations, as the pandemic evolves (Zhang \& Holmes 2020). In this scenario, it is expected that diverse signatures of viral variants spread among different populations in the world. Recently, thousands of GenBank sequences from SARS-CoV19 available at the NCBI virus database were trackable by region, suggesting that the transmission occurred mainly through clonal events due to clustering of the available sequences (Chen et al. 2020; Kupferschmidt

2020) (https://www.ncbi.nlm.nih.gov/labs/virus/vssi/\#/virus?SeqType_s=Protein\&VirusLineage_ss=S ARS-CoV-2,\%20taxid:2697049). As a proof of concept, in the early beginning of the outbreak in China, sequencing the virus from nine patients from Wuhan in China revealed $99.9 \%$ similarity among samples. That finding suggests $2019-\mathrm{nCoV}$ originated from one source within a very short time, supporting clonality of spreading (Lu et al. 2020). In this study, we characterized the early mutational events across 50 illumina high-quality datasets publicly available on the sequence read archive repository. 30 out of 50 samples $(60 \%)$ contained at least a single founder variant and most of the variants across samples are missense (over 63\%). SARS-CoV-2 founder variants in 
80

81

82

83

84

85

86

87

88

89

90

91

92

93

94

95

96

97

98

99

100

101

102

103

104

105

106

107

108

109

110

111

112

113

114

115

116

117

118

119

Washington State, USA (USA-WA) are dissimilar to Australian SARS-CoV-2 founder variants, which were found to be heterogeneous. However, a mutational signature from USA mutations was found in an Australian sample, suggesting a world-wide spread of this molecular signature consisting of five-point variants. Remarkably, mutations in the helicase and ORF 1ab proteins of the virus were found more frequently than others, suggesting that these regions continue to actively evolve. As proof of the latter, a single nucleotide polymorphism (SNP) in an Australian sample causes a bona-fide stop codon in the helicase protein. As genetic drift prompts the mutational spectrum of the virus, we recommend frequently sequencing the viral pool in every country to detect the founder events relevant for SARS-CoV-2 testing in each population.

\section{Materials \& Methods}

\section{Data Collection}

Raw illumina sequencing data were downloaded from the following NCBI SRA BioProjects: SRA: PRJNA601736 (Chinese datasets), SRA: PRJNA603194 (Chinese dataset) (Wu et al. 2020b), SRA: PRJNA605907 (Chinese datasets) (Shen et al. 2020), SRA: PRJNA607948 (USAWisconsin State datasets), SRA: PRJNA608651 (Nepal dataset), SRA: PRJNA610428 (USAWashington State datasets), SRA: PRJNA612578 (USA-San-Diego dataset), SRA: PRJNA231221 (USA-Washington State dataset) (Sichtig et al. 2019), SRA: PRJNA613958 (Australian-Victoria datasets), SRA: PRJNA231221 (USA-Maryland State dataset), and SRA: PRJNA614995 (USAUtah datasets). All illumina SRA accessions until March 27, 2020 are depicted in Supplementary Table 1, sheet 1. Illumina SRA accessions until April 22, 2020 are available in https://github.com/cfarkas/SARS-CoV-2 illumina analysis and were obtained from SARS-CoV2 resource at GenBank: https://www.ncbi.nlm.nih.gov/genbank/sars-cov-2-seqs/.

\section{Data processing}

Primer sequences were aligned with bowtie2 aligner (v2.2.6) (Langmead \& Salzberg 2012) against SARS-CoV-2 reference genome NC_045512.2 (https://www.ncbi.nlm.nih.gov/nuccore/NC_045512), using the following parameters: -D 20 -R 3 -N 0 -L 20 -i S,1,0.50. Illumina Raw reads from whole genome sequencing and amplicon sequencing were trimmed by using fastp tool in default mode (Chen et al. 2018) and aligned by using Minimap2 aligner using the preset -ax sr against SARS-CoV-2 reference genome (Li 2018). GenBank fasta sequences form Asia, Europe and North America were also aligned by using minimap2 with the same preset. Samtools v1.9 (using htslib v1.9) (Li et al. 2009) was used to sort sam files and index bam files. bcftools v1.9 (part of the samtools framework) was used to obtain depth of coverage in each aligned sample. For variant calling in illumina samples, Strelka2 variant caller was employed in each bam dataset by invoking GermlineWorkflow and outputted variants were filtered by using the "PASS" criteria (Kim et al. 2018). Variants from next generation sequencing reads and GenBank alignments were also called with bcftools mpileup with the 
120 following parameters: -B -C 50 -d 250. To obtain founder mutations, filtering of called variants

121

122

123

124

125

126

127

128

129

130

131

132

133

134

135

136

137

138

139

140

141

142

143

144

145

146

147

148

149

150

151

152

153

154

155

156

157

158

159

was performed with bcftools filter, considering variants only with Mann-Whitney $U$ test of read position bias over 0.1 and the number of high-quality reference alleles divided by high-quality alternate alleles over 0.3 . All commands to obtain these computational steps are publicly available at https://github.com/cfarkas/SARS-CoV-2_illumina_analysis.

\section{SNVs consequences and classification}

All SNP and INDELs consequences were assessed in each sample by using snippy haploid variant calling and core genome alignment pipeline: https://github.com/tseemann/snippy. Also, Variant effect annotation tool, employing the variant effect predictor algorithm (VEP) was employed to assess functional effects of variants on SARS-CoV-2 transcripts.(Hinrichs et al. 2016; McLaren et al. 2016).

\section{Construction of multiple sequence alignments with GenBank sequences and phylogenetic} tree inference

1599 SAR2-CoV-2 GenBank datasets from Asia $(n=190)$, Europe $(n=40)$ and North America $(n=1359)$ were downloaded on April 22, 2020 from NCBI virus database (https://www.ncbi.nlm.nih.gov/labs/virus/vssi/\#/) using as query "Severe acute respiratory syndrome coronavirus 2 (SARS-CoV-2), taxid:2697049". All sequences are publicly available at https://github.com/cfarkas/SARS-CoV-2 illumina analysis. Merged sequences were also aligned by using MAFFT multiple sequence alignment program version 7.271 (Katoh \& Standley 2013) using the -reorder flag. Fasttree version 2.1 was used to infer an approximately-maximumlikelihood phylogenetic tree from the aligned sequences in fasta format by using heuristic neighbor-joining clustering method (Price et al. 2010). Visualization and editing of the phylogenetic tree were perfomed by using Interactive Tree of Life server (iTOL), collapsing all clades whose average branch length distance was below 0.0002 (Letunic \& Bork 2019).

\section{Primer List obtention}

CDC primers currently in use (April 2020) were obtained from https://www.cdc.gov/coronavirus/2019-ncov/lab/rt-pcr-panel-primer-probes.html, generated by the Division of Viral Diseases, National Center for Immunization and Respiratory Diseases, Centers for Disease Control and Prevention, Atlanta, GA, USA. University of Hong-Kong primers currently in use from the were obtained from https://www.who.int/docs/defaultsource/coronaviruse/peiris-protocol-16-1-20.pdf?sfvrsn=aflaac73_4 and Institute Pasteur primers currently in use were obtained based on the first sequences of SARS-CoV-2 made available on the GISAID database (Shu \& McCauley 2017) on January 11, 2020, available here: https://www.who.int/docs/default-source/coronaviruse/real-time-rt-pcr-assays-for-the-detectionof-sars-cov-2-institut-pasteur-paris.pdf?sfvrsn=3662fcb6_2. We also included literature-based primers (Kim et al. 2020) and ten primer-BLAST (Ye et al. 2012) designed primers against SARSCoV-2 reference genome NC_045512.2. 


\section{Results}

162

Inspection of variants reveals well-defined signatures with founder effects across sequenced samples.

165

We aimed to call variants of SARS-CoV-2 datasets sequenced with the Illumina technology, due

167

168

169

170

171

172

173

174

175

176

177

178

179

180

181

182

183

184

185

186

187

188

189

190

191

192

193

194

195

196

197

198

199 to its depth and sequencing quality, in terms of error rate (Nielsen et al. 2011). As of March 27, 2020 we obtained 282 accession numbers for SARS-CoV-2, from the sequence read archive, containing 27 illumina datasets. By searching in Sequence Read Archive repository (SRA) we added 24 more datasets yielding in total 51 illumina raw datasets to analyse (see Supplementary Table 1, sheet 1 and 2). From this list, we excluded the Chinese Sample nCoV5 (SRR11059943) due to large gaps in genome coverage, as explained in (Shen et al. 2020) and we subsequently worked with these illumina datasets. We aligned each fastq reads against the SARS-CoV-2 reference genome NC_045512.2, corresponding to the initial isolate Wuhan-Hu-1. We checked coverage of each sample by using the Integrative Genomics Viewer (IGV) tool (Robinson et al. 2011) and samtools (Supplementary Table 1, sheet 2). Variant calling in each sample by using Strelka2 reveals a diverse number of variants in each sample, yielding 137 single nucleotide polymorphisms (SNVs) and 9 indels (see Figure 1A, Supplementary Table 1, sheet 3). Founder variants were obtained by doing variant calling with bcftools after strict filtering (see Supplementary Table 1, sheet 2). Remarkably, thirteen out of fourteen datasets from the USAWashington State study (hereafter referred as USA-WA) displayed variants presenting a defined variant signature, consisting in a core of five founder variants at positions 8782, 17747, 17858, 18060 and 28144 in the SARS-CoV-2 reference genome, also detected within the 137 SNVs from the next generation sequencing datasets (see Figure 1B, Supplementary Table 1, sheet 2 and sheet 3). Mutational landscape analysis of SARS-CoV-2 samples in Australia (Australia-Victoria samples, hereafter Australia-VIC) demonstrated that these samples were clearly heterogeneous, displaying a variety of founder mutations per sample but also shared variants were observed within samples. One variant (position 26144) is present in 5/11 Australia-VIC samples and variants 8782 and 28144 from USA-WA signature are also present in 3/11 Australian samples (see Figure 1B, Supplementary Table 1, sheet 2). Notably, one Australian-VIC sample (SRR11397717) displayed the same five-point variant signature of USA-WA samples, two samples contain the same variant signature presenting one deletion (SRR11397715 and SRR11397716) and one novel signature (SRR11397728) presents a SNP that creates a stop codon (see Figure 1B, respectively). All of these called variants present mutant allele frequencies near or equal to $100 \%$, evidenced in the number of mutant_alleles / reference_alleles (see mutant allele frequency in Supplementary Table 1, sheet 4 and 5, respectively) easily visualized in the aligned bam files (see Figure 1C from USA-WA and Australian-VIC samples, respectively). These analyses suggest that these variants were already spread in the infected population in the early days of the outbreak, they are not restricted by country and that they will continue to spread along with the growing cases. To support 
200 the latter, as of April 22, 2020 we downloaded 1599 GenBank sequences of SARS-CoV-2 from 201 Asia, Europe and North America origin, respectively and we aligned them with the SARS-CoV-2 202 reference genome. A phylogenetic tree was constructed from all genbank sequences and depict a 203 mixed clustering of sequences between Asia, Europe and North America, supporting the existance 204 of different viral signatures. (Supplementary Figure 1). Variant calling from these alignment 205 reveals the substantial presence of USA-WA signature in North America sequences, with allele 206 frequencies (AF) ranging 33-39\% (see Figure 1D, and Supplementary Table 1, sheet 6). Variants 2078782 and 28144 from USA-WA signature are also present in Asia and Europe, suggesting these 208 two variants arises in the beginning of the pandemic and spread worldwide. Thus, the USA-WA 209 signature is likely widespread among SARS-CoV-2 infections in USA due to founder effect. Also, 210 in North America GenBank sequences, six more variants were detected with similar allele 211 frequencies as reported for USA-WA variants. A summary of the USA-WA mutational signature 212 is depicted in Table 1. Less founder variants with lower allele frequencies are present in Asian 213 samples suggesting high clonality of the original strain of SARS-CoV-2. Conversely, in Europe 214 sixteen founder variants with higher allele frequencies were found, supporting SARS-CoV-2 215 evolves as the pandemic spread. Overall, missense GenBank variants equals or surpasses 216 synonymous variants in ORF1ab, Nucleocapsid (N), ORF3a, ORF8 and surface glycoprotein (S) 217 (see Figure 1E, Supplementary Table 1, sheet 7). Thus, as the pandemic evolves, successful 218 molecular adaptations in SARS-CoV-2 also occurred as is presented in the different viral 219 signatures.

220

\section{Classification of next-generation sequencing variants}

To see if the latter observations are replicated in next generation sequencing datasets, we aimed to characterize in depth USA-WA and Australia-VIC variants as performed with founder variants from GenBank sequences. In agreement with the latter, classification of variants performed by snippy tool analysis reveals that most variants in USA-WA and Australia-VIC are preferentially missense (63\% for USA-WA samples and $74 \%$ for Australia-VIC samples, respectively) rather

229

230

231

232

233

234

235

236

237

238

239 than synonymous (see Figure 2A, Supplementary Table 1, sheets 4 and 5, respectively). Focusing on missense variants of USA-WA samples, the most recurrent mutations occurred in the polypeptide ORF1ab (encoding a viral helicase and 3 '-to-5' exonuclease) and ORF8 protein, accounting for the $81 \%$ of missense variants in the USA-WA signature (see Figure 2B, Supplementary Table 1, sheet 4). In the case of Australia-VIC missense variants, the scenario is more complex, due to the heterogeneity of the signatures. Mutations in the orfla polyprotein, ORF3a protein, helicase and surface glycoprotein account for $69 \%$ of the missense variants present in the Australia-VIC signatures. Importantly, two Australian samples (SRR11397715 and SRR11397716) present the same mutational profiling with deletions in the stem loop of the virus and notably, one sample (SRR11397728) presents a SNP that creates a stop codon in the helicase protein (see Supplementary Table 1, sheet 5). Since every USA-WA and Australia-VIC sample that presents variants in the helicase gene contains at least one missense variant, this evidence 
240

241

242

243

244

245

246

247

248

249

250

251

252

253

254

255

256

257

258

259

260

261

262

263

264

265

266

267

268

269

270

271

272

273

274

275

276

277

278

279

strongly suggests that this gene is located in an actively evolving region from SARS-CoV-2 and it will likely continue to evolve as the pandemic spreads.

\section{SNPs in SARS-CoV-2 may diminish efficiency of RT-qPCR testing.}

The Centers for Disease Control and Prevention primer list, consists of three primer sets designed against the ORF9 structural protein (nucleocapsid phosphoprotein), each one with a fluorescent probe for reverse transcriptase quantitative PCR. We investigated if these primers hybridized at positions that fall within the variants reported herein from the 50 next-generation sequencing datasets. We aligned these primer sequences with SARS-CoV-2 reference genome and the 50 analyzed samples. We found two Australian clonal samples (SRR11397719 and SRR11397721) presenting one founder synonymous SNP (position 28688) that occurs within the 2019_nCoV_N3_Forward_Primer hybridization region. In the first sample, we also found an SNP with low allele frequency that falls into the 2019-nCoV_N1_Reverse_Primer (see Figure 3). We also challenged a list of primers available from literature (16), primers currently used for the Institute Pasteur, Hong Kong University and ten primers obtained from primer-blast against merged GenBank variants including variants from the next-generation sequencing datasets employed here. Of these, CDC primer set 2019-nCoV_N3 forward and reverse along with its probe were discarded again, due to potential reduced efficiency during priming (see Supplementary Table 1, sheet 8). Similarly, sets 1, 2, 3 and 8 from primer-blast, were discarded. Conversely, primers used by the Pasteur Institute and Hong-Kong university passed the filter, respectively. Thus, increasing variation in SARS-CoV-2 can confound quantitative RT-qPCR in the future depending on the primer design.

\section{Discussion}

In this study, we have analyzed early mutational events occurring in SARS-CoV-2 illumina wholegenome sequencing samples from different populations (USA, Australia and China) and we compared these results with variants observed in submitted GenBank sequences in NCBI viral portal up until April 22, 2020. As already reported with HIV and Chikungunya outbreaks, the founder effect of five-point variants was observed in almost all USA-WA samples obtained by next-generation sequencing. These mutations also have high allele frequencies (around 33-39\%) in SARS-CoV-2 GenBank sequences from USA origin (Foley et al. 2000; Tsetsarkin et al. 2011). These variant signatures are likely to be overrepresented among Washington State infections and USA infections overall, if not globally. Supporting the latter, the alignment of 1359 SARS-CoV2 submitted sequences to NCBI virus from North America (April 22, 2020) shows allele frequencies of 33-39\% of these five SNPs across samples worldwide. These SNPs cause missense mutations in helicase, 3'-to-5' exonuclease and ORF8 proteins. In the case of Victoria samples from Australia, founder variants from one up to eleven SNPs were found. In the early beginning

Peer] reviewing PDF | (2020:04:47559:2:0:NEW 6 May 2020) 
280

281

282

283

284

285

286

287

288

289

290

291

292

293

294

295

296

297

298

299

300

301

302

303

304

305

306

307

308

309

310

311

312

313

314

315

316

317

318

319

of the outbreak in Wuhan city (between December 18-29, 2019), one to four mutations arose in the virus per patient (Shen et al. 2020), arguing that the number of fixed mutations in the world population is rapidly increasing where the infection has spread. Importantly, one Australian sample sequenced by next generation sequencing presented the USA-WA signature, suggesting that this signature is already propagated with the worldwide pandemic. Consistent with the latter, real-time tracking of pathogen evolution and phylogenetic analysis provided from the Nextstrain initiative demonstrated dissemination of SARS-CoV-2 viral signatures from USA to Australia and Europe in early February 2020 (Hadfield et al. 2018). Clonal mutational events within Australian samples were also observed and probably are widespread in the region. One interesting feature from USAWA signature are missense variants occurring in the helicase gene (ORF1ab). RNA helicases display various functions in genome replication, they have even been proposed as a therapeutic target to inhibit coronaviruses among other viruses with small molecules (Briguglio et al. 2011), thus mutations evoked by these variants could make drug targeting this protein more difficult in the future. Also, as genetic drift is allowing SARS-CoV-2 to evolve as the pandemic continues, the amplification efficiency of quantitative RT-qPCR tests may be compromised since a single mutational even in the middle of a primer sequence can be detrimental for PCR efficiency (Bru et al. 2008), potentially contributing to false negative results in COVID-19 testing. For this issue, we provided a way to compute the latter, by merging all variant sites called across studied samples and by intersecting them across primer sets available both in the literature and currently in use in viral testing kits. As new mutations can be spread depending on the founder effect, we firmly urge that primer sets for clinical testing should be tested in this way continuously, according to the current mutations found at the particular time and in the specific population which needs to be diagnosed with SARS-CoV-2 infection.

\section{Conclusions}

We describe here the early mutational events in SARS-CoV-2 virus by analyzing sequencing samples from China, USA, Australia and GenBank sequences submitted between March 27 and April 22, 2020. SARS-CoV-2 variants from the USA display five-point mutations with clonal (founder) patterns of spreading at a considerably high frequency among samples. The latter was verified by sequence analysis of SARS-CoV-2 sequences submitted to GenBank, since these fivepoint mutations displayed alleles frequencies of 33-39\% among all USA GenBank SARS-CoV-2 sequences $(n=1359)$. SARS-CoV-2 Australian variants were heterogeneous, but still, clonal events were found including one sample presenting the USA-WA signature, implying worldwide spreading of this signature. The efficiency of RT-qPCR testing can be potentially affected by founder variants, since several SNPs affecting one of three primers sets currently used in COVID19 testing has been found. By the time of this publication, the available data could change the conclusions presented in this manuscript as a result of further viral variants arising.

Peer) reviewing PDF | (2020:04:47559:2:0:NEW 6 May 2020) 
321

322

323

324

325

326

327

328

329

330

331

332

333

334

335

336

337

338

339

340

341

342

343

344

345

346

347

348

349

350

351

352

353

354

355

356

357

358

359

360

361

362

363

364

365

366

\section{Acknowledgements}

We thank valuable discussions from Convenio de colaboración diagnóstico y otras actividades relativas al COVID-19. Agencia Nacional de Investigacion y Desarrollo, ANID, PROYECTO COVID ID18I10261.

\section{References}

Briguglio I, Piras S, Corona P, and Carta A. 2011. Inhibition of RNA Helicases of ssRNA(+) Virus Belonging to Flaviviridae, Coronaviridae and Picornaviridae Families. Int $J$ Med Chem 2011:213135. 10.1155/2011/213135

Bru D, Martin-Laurent F, and Philippot L. 2008. Quantification of the detrimental effect of a single primer-template mismatch by real-time PCR using the 16S rRNA gene as an example. Appl Environ Microbiol 74:1660-1663. 10.1128/AEM.02403-07

Chen Q, Allot A, and Lu Z. 2020. Keep up with the latest coronavirus research. Nature 579:193. 10.1038/d41586-020-00694-1

Chen S, Zhou Y, Chen Y, and Gu J. 2018. fastp: an ultra-fast all-in-one FASTQ preprocessor. Bioinformatics 34:i884-i890. 10.1093/bioinformatics/bty560

Coutard B, Valle C, de Lamballerie X, Canard B, Seidah NG, and Decroly E. 2020. The spike glycoprotein of the new coronavirus 2019-nCoV contains a furin-like cleavage site absent in CoV of the same clade. Antiviral Res 176:104742. 10.1016/j.antiviral.2020.104742

de Wit E, van Doremalen N, Falzarano D, and Munster VJ. 2016. SARS and MERS: recent insights into emerging coronaviruses. Nat Rev Microbiol 14:523-534. 10.1038/nrmicro.2016.81

Foley B, Pan H, Buchbinder S, and Delwart EL. 2000. Apparent founder effect during the early years of the San Francisco HIV type 1 epidemic (1978-1979). AIDS Res Hum Retroviruses 16:1463-1469. 10.1089/088922200750005985

Hadfield J, Megill C, Bell SM, Huddleston J, Potter B, Callender C, Sagulenko P, Bedford T, and Neher RA. 2018. Nextstrain: real-time tracking of pathogen evolution. Bioinformatics 34:4121-4123. 10.1093/bioinformatics/bty407

Hinrichs AS, Raney BJ, Speir ML, Rhead B, Casper J, Karolchik D, Kuhn RM, Rosenbloom KR, Zweig AS, Haussler D, and Kent WJ. 2016. UCSC Data Integrator and Variant Annotation Integrator. Bioinformatics 32:1430-1432. 10.1093/bioinformatics/btv766

Hu D, Zhu C, Ai L, He T, Wang Y, Ye F, Yang L, Ding C, Zhu X, Lv R, Zhu J, Hassan B, Feng Y, Tan W, and Wang C. 2018. Genomic characterization and infectivity of a novel SARSlike coronavirus in Chinese bats. Emerg Microbes Infect 7:154. 10.1038/s41426-0180155-5

Katoh K, and Standley DM. 2013. MAFFT multiple sequence alignment software version 7: improvements in performance and usability. Mol Biol Evol 30:772-780. 10.1093/molbev/mst010

Kim JM, Chung YS, Jo HJ, Lee NJ, Kim MS, Woo SH, Park S, Kim JW, Kim HM, and Han MG. 2020. Identification of Coronavirus Isolated from a Patient in Korea with COVID-19. Osong Public Health Res Perspect 11:3-7. 10.24171/j.phrp.2020.11.1.02

Kim S, Scheffler K, Halpern AL, Bekritsky MA, Noh E, Kallberg M, Chen X, Kim Y, Beyter D, Krusche $P$, and Saunders CT. 2018. Strelka2: fast and accurate calling of germline and somatic variants. Nat Methods 15:591-594. 10.1038/s41592-018-0051-x 
367

368

369

370

371

372

373

374

375

376

377

378

379

380

381

382

383

384

385

386

387

388

389

390

391

392

393

394

395

396

397

398

399

400

401

402

403

404

405

406

407

408

409

410

411

412

413

414

415

416

Kupferschmidt K. 2020. Genome analyses help track coronavirus' moves. Science 367:11761177. 10.1126/science.367.6483.1176

Langmead B, and Salzberg SL. 2012. Fast gapped-read alignment with Bowtie 2. Nat Methods 9:357-359. 10.1038/nmeth. 1923

Letunic I, and Bork P. 2019. Interactive Tree Of Life (iTOL) v4: recent updates and new developments. Nucleic Acids Res 47:W256-W259. 10.1093/nar/gkz239

Li H. 2018. Minimap2: pairwise alignment for nucleotide sequences. Bioinformatics 34:30943100. 10.1093/bioinformatics/bty191

Li H, Handsaker B, Wysoker A, Fennell T, Ruan J, Homer N, Marth G, Abecasis G, Durbin R, and Genome Project Data Processing S. 2009. The Sequence Alignment/Map format and SAMtools. Bioinformatics 25:2078-2079. 10.1093/bioinformatics/btp352

Lu R, Zhao X, Li J, Niu P, Yang B, Wu H, Wang W, Song H, Huang B, Zhu N, Bi Y, Ma X, Zhan F, Wang L, Hu T, Zhou H, Hu Z, Zhou W, Zhao L, Chen J, Meng Y, Wang J, Lin Y, Yuan J, Xie Z, Ma J, Liu WJ, Wang D, Xu W, Holmes EC, Gao GF, Wu G, Chen W, Shi W, and Tan W. 2020. Genomic characterisation and epidemiology of 2019 novel coronavirus: implications for virus origins and receptor binding. Lancet 395:565-574. 10.1016/S0140-6736(20)30251-8

McLaren W, Gil L, Hunt SE, Riat HS, Ritchie GR, Thormann A, Flicek P, and Cunningham F. 2016. The Ensembl Variant Effect Predictor. Genome Biol 17:122. 10.1186/s13059-0160974-4

Nielsen R, Paul JS, Albrechtsen A, and Song YS. 2011. Genotype and SNP calling from nextgeneration sequencing data. Nat Rev Genet 12:443-451. 10.1038/nrg2986

Price MN, Dehal PS, and Arkin AP. 2010. FastTree 2--approximately maximum-likelihood trees for large alignments. PLoS One 5:e9490. 10.1371/journal.pone.0009490

Robinson JT, Thorvaldsdottir H, Winckler W, Guttman M, Lander ES, Getz G, and Mesirov JP. 2011. Integrative genomics viewer. Nat Biotechnol 29:24-26. 10.1038/nbt.1754

Shen Z, Xiao Y, Kang L, Ma W, Shi L, Zhang L, Zhou Z, Yang J, Zhong J, Yang D, Guo L, Zhang G, Li H, Xu Y, Chen M, Gao Z, Wang J, Ren L, and Li M. 2020. Genomic diversity of SARS-CoV-2 in Coronavirus Disease 2019 patients. Clin Infect Dis. 10.1093/cid/ciaa203

Shu Y, and McCauley J. 2017. GISAID: Global initiative on sharing all influenza data - from vision to reality. Euro Surveill 22. 10.2807/1560-7917.ES.2017.22.13.30494

Sichtig H, Minogue T, Yan Y, Stefan C, Hall A, Tallon L, Sadzewicz L, Nadendla S, Klimke W, Hatcher E, Shumway M, Aldea DL, Allen J, Koehler J, Slezak T, Lovell S, Schoepp R, and Scherf U. 2019. FDA-ARGOS is a database with public quality-controlled reference genomes for diagnostic use and regulatory science. Nat Commun 10:3313. 10.1038/s41467-019-11306-6

Tsetsarkin KA, Chen R, Leal G, Forrester N, Higgs S, Huang J, and Weaver SC. 2011. Chikungunya virus emergence is constrained in Asia by lineage-specific adaptive landscapes. Proc Natl Acad Sci U S A 108:7872-7877. 10.1073/pnas.1018344108

Wilder-Smith A, Chiew CJ, and Lee VJ. 2020. Can we contain the COVID-19 outbreak with the same measures as for SARS? Lancet Infect Dis. 10.1016/S1473-3099(20)30129-8

Wu A, Peng Y, Huang B, Ding X, Wang X, Niu P, Meng J, Zhu Z, Zhang Z, Wang J, Sheng J, Quan L, Xia Z, Tan W, Cheng G, and Jiang T. 2020a. Genome Composition and Divergence of the Novel Coronavirus (2019-nCoV) Originating in China. Cell Host Microbe 27:325-328. 10.1016/j.chom.2020.02.001

Wu F, Zhao S, Yu B, Chen YM, Wang W, Song ZG, Hu Y, Tao ZW, Tian JH, Pei YY, Yuan ML, Zhang YL, Dai FH, Liu Y, Wang QM, Zheng JJ, Xu L, Holmes EC, and Zhang YZ. $2020 \mathrm{~b}$. A new coronavirus associated with human respiratory disease in China. Nature 579:265-269. 10.1038/s41586-020-2008-3

Peer] reviewing PDF | (2020:04:47559:2:0:NEW 6 May 2020) 
417 Ye J, Coulouris G, Zaretskaya I, Cutcutache I, Rozen S, and Madden TL. 2012. Primer-BLAST:

$418 \quad$ a tool to design target-specific primers for polymerase chain reaction. BMC

419 Bioinformatics 13:134. 10.1186/1471-2105-13-134

420 Zhang T, Wu Q, and Zhang Z. 2020. Probable Pangolin Origin of SARS-CoV-2 Associated with

421 the COVID-19 Outbreak. Curr Biol. 10.1016/j.cub.2020.03.022

422 Zhang YZ, and Holmes EC. 2020. A Genomic Perspective on the Origin and Emergence of

423

424 SARS-CoV-2. Cell 181:223-227. 10.1016/j.cell.2020.03.035 


\section{Figure 1}

Inspection of variants reveals well-defined signatures with founder effect across sequenced samples

A) (Upper) Plot of all merged variants from NGS datasets $(n=50)$ depicting single nucleotide variants (black dots) and indels (black squares) along SARS-CoV-2 nucleotide positions. (Lower) Snapshot of SARS-CoV-2 ORFs and receptor binding domains. User track denotes merged NGS variants across ORFs. B) Plot of founder variants sorted by country (China=red, USA=blue and Australia=green) and by the number of variants, from left to right. Deletions and stop codons are framed with black rectangles. C) Top IGV screenshots of coverage from USA samples $(n=13)$ aligned against SARS-CoV-2 reference genome. Founder variants are depicted with colored lines. Bottom IGV screenshots of coverage from Australian samples $(n=11)$ aligned against SARS-CoV-2 reference genome. Founder variants are depicted with colored lines. D) Allele frequency (plotted as percentage) of founder variants collected from GenBank SARS-CoV-2 sequence alignments from Asia $(n=270)$, Europe $(n=50)$ and North America ( $n=1359$ ). Asterisks denotes common founder variants in the three regions, part of the USA-WA signature (8782, 28144). E) (Upper) Variant consequence classification of GenBank founder variants obtained with the variant annotator integrator tool. Missense and synonymous variant consequences per ORF are denoted with red grey bars, respectively. (Lower) Schematic diagram of the general genetic composition of SARS-Cov-2. Colored boxes correspond to main genes and white boxes to smaller ORFs. 
A

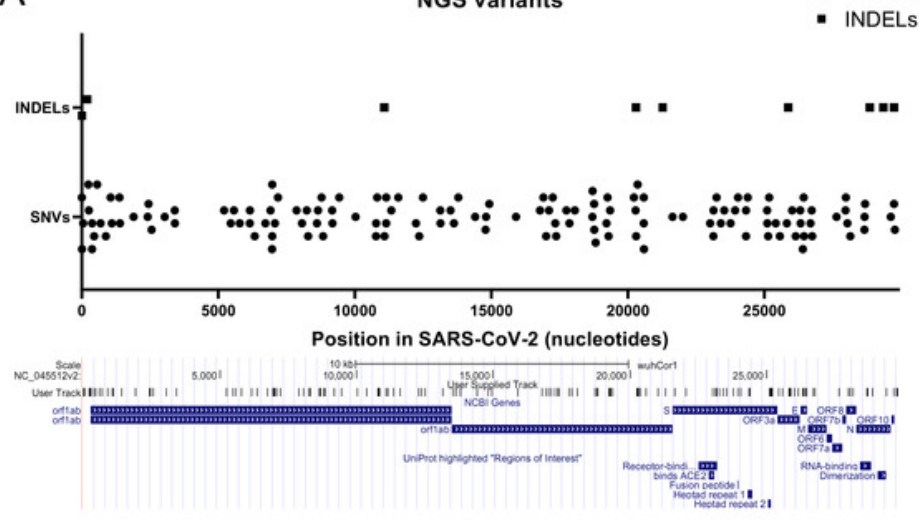

B

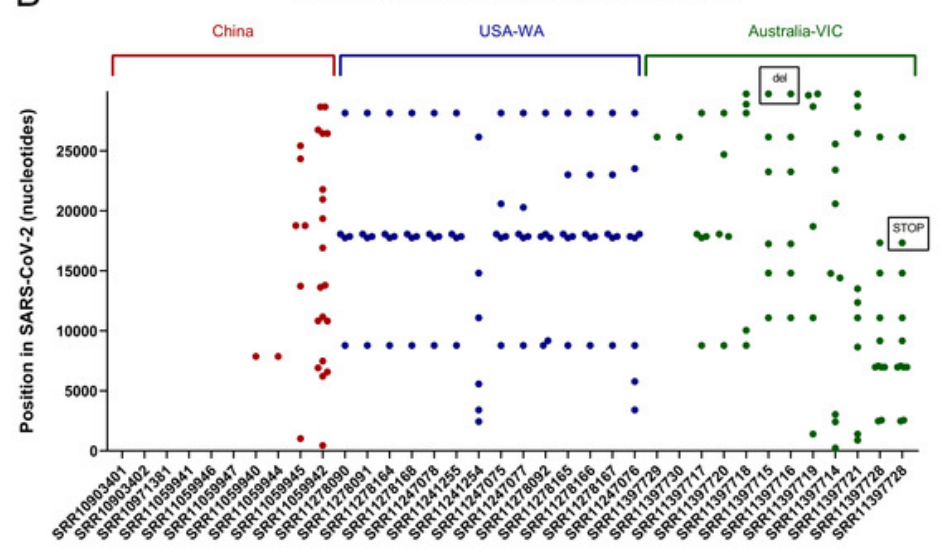

Samples
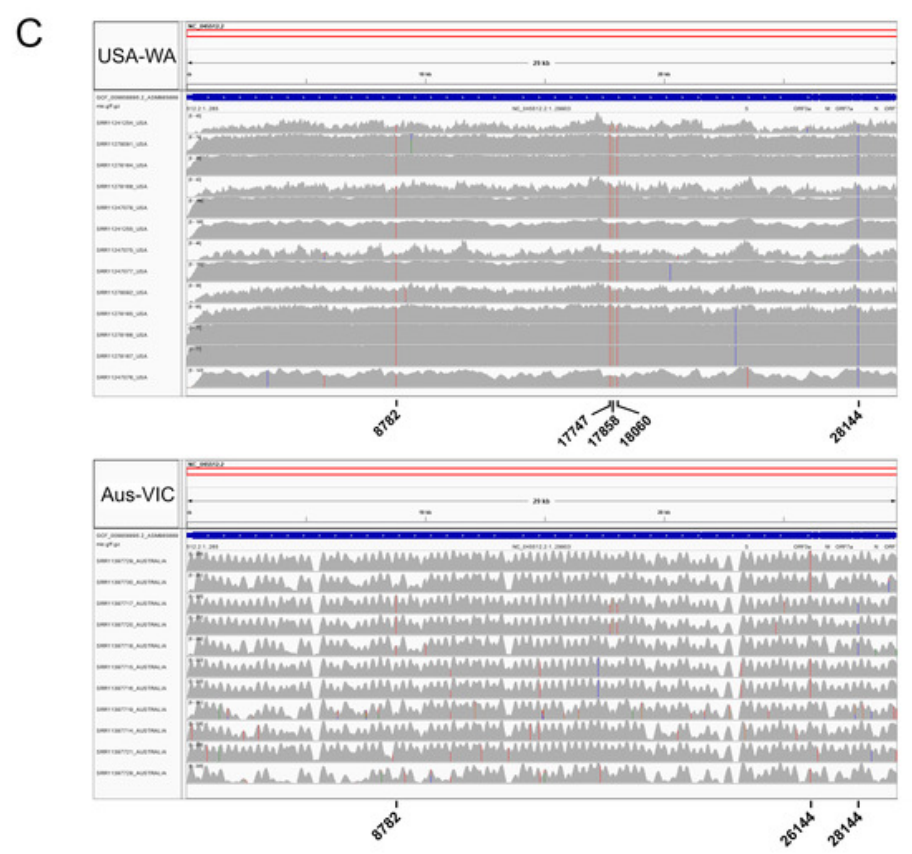

E

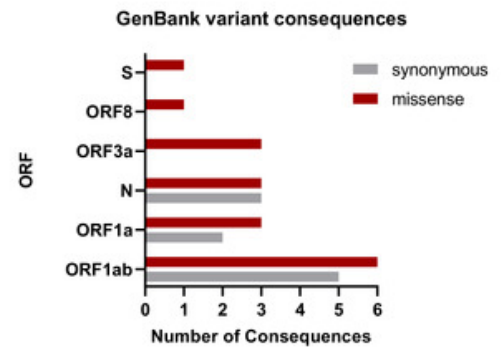

GenBank Sequences (April 22, 2020)

Asia: 190

Europe: 50

North_America: 1359
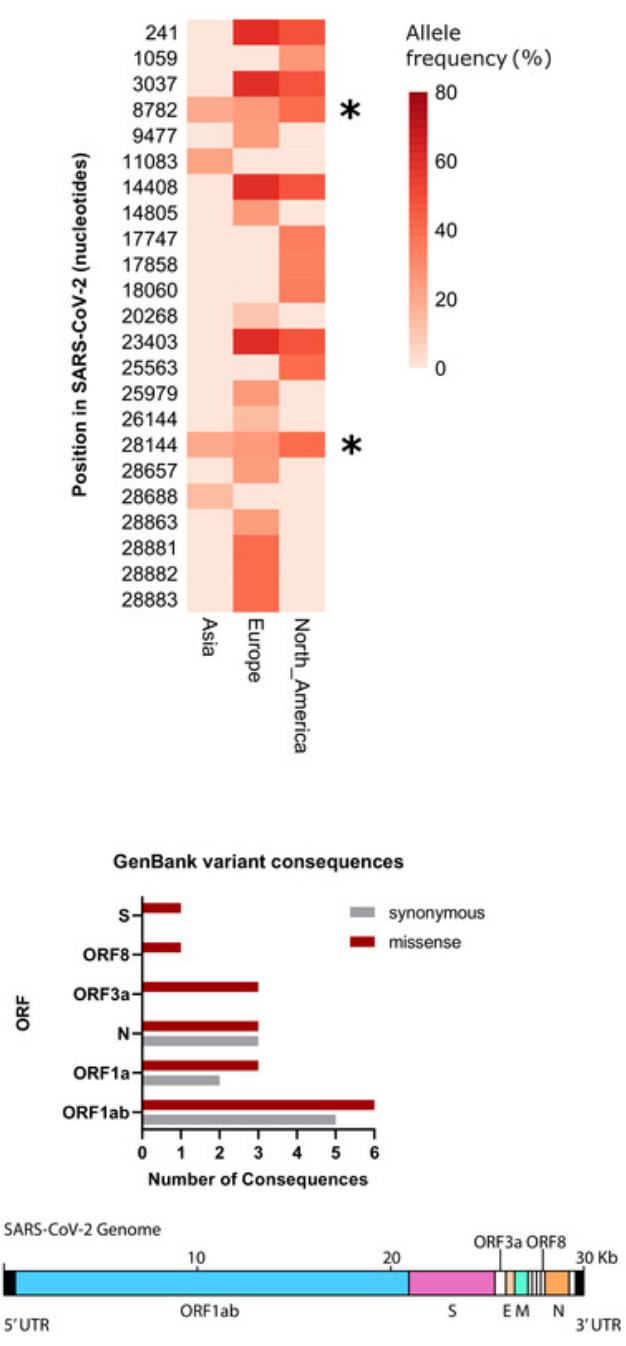


\section{Figure 2}

Classification of SARS-CoV-2 variants from next-generation sequencing datasets

A) (Left) Ratio of Synonymous / missense variants across USA samples. Missense variants are denoted in black and Synonymous variants are depicted in grey $\mathrm{N}$ indicates the total number of classified variants. (Right) Same as left for Australian samples. B) (Left) Missense variants classification from USA samples across SARS-CoV-2 genes. Each color indicates a different gene stated in legend (at right of the pie charts). (Right) Same as left from Australian samples.

A

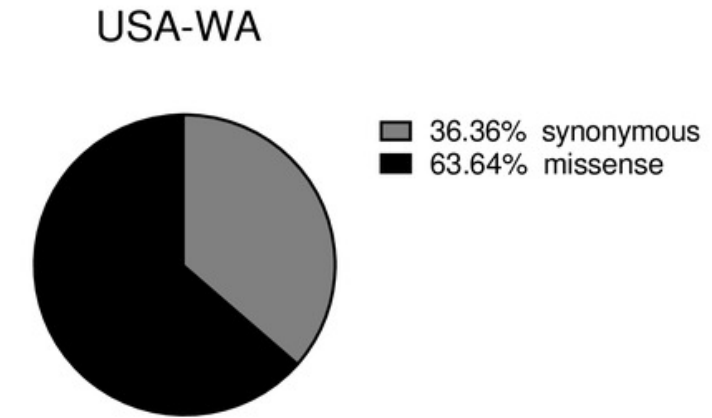

Total $=77$
Australia-VIC

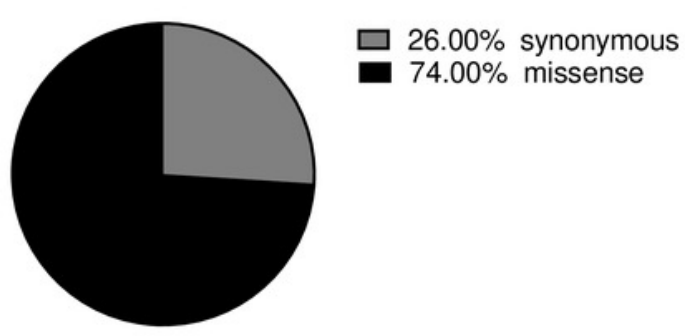

Total $=50$

B

USA: Variants per gene

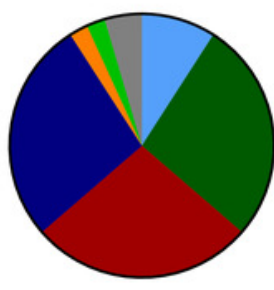

Total $=\mathbf{4 4}$
27.27\% helicase

$27.27 \%$ 3'-to-5' exonuclease

$27.27 \%$ ORF8 protein

$\square 9.09 \%$ orf1a polyprotein

$\square 4.55 \%$ surface glycoprotein

$\square 2.27 \%$ RNA-dependent RNA pol

$\square 2.27 \%$ ORF3a protein
Aus: Variants per gene

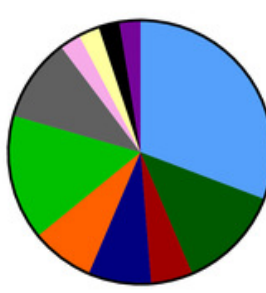

Total $=39$ $\square 30.77 \%$ orf1a polyprotein

$\square 15.38 \%$ ORF3a protein

$\square 12.82 \%$ helicase

$10.26 \%$ surface glycoprotein

- $7.69 \%$ ORF8 protein

$\square 7.69 \%$ RNA-dependent RNA pol

$\square$ 5.13\% 3'-to-5' exonuclease

$\square 2.56 \%$ nucleocapsid phosphoprotein

$\square 2.56 \%$ ORF10

- $2.56 \%$ endoRNAse

$\square 2.56 \%$ envelope protein 
Figure 3

SNPs in SARS-CoV-2 may diminish efficiency of RT-qPCR testing

A) Top IGV screenshots of coverage from Australian sample SRR11397719 aligned against SARS-CoV-2 reference genome. A low allele frequency variant is depicted in red (T). Primers tracks are denoted at the top of the screenshot along with SARS-CoV- 2 gff gene models. Bottom Same as Top for sample SRR11397719 denoting a founder variant in blue. 
A
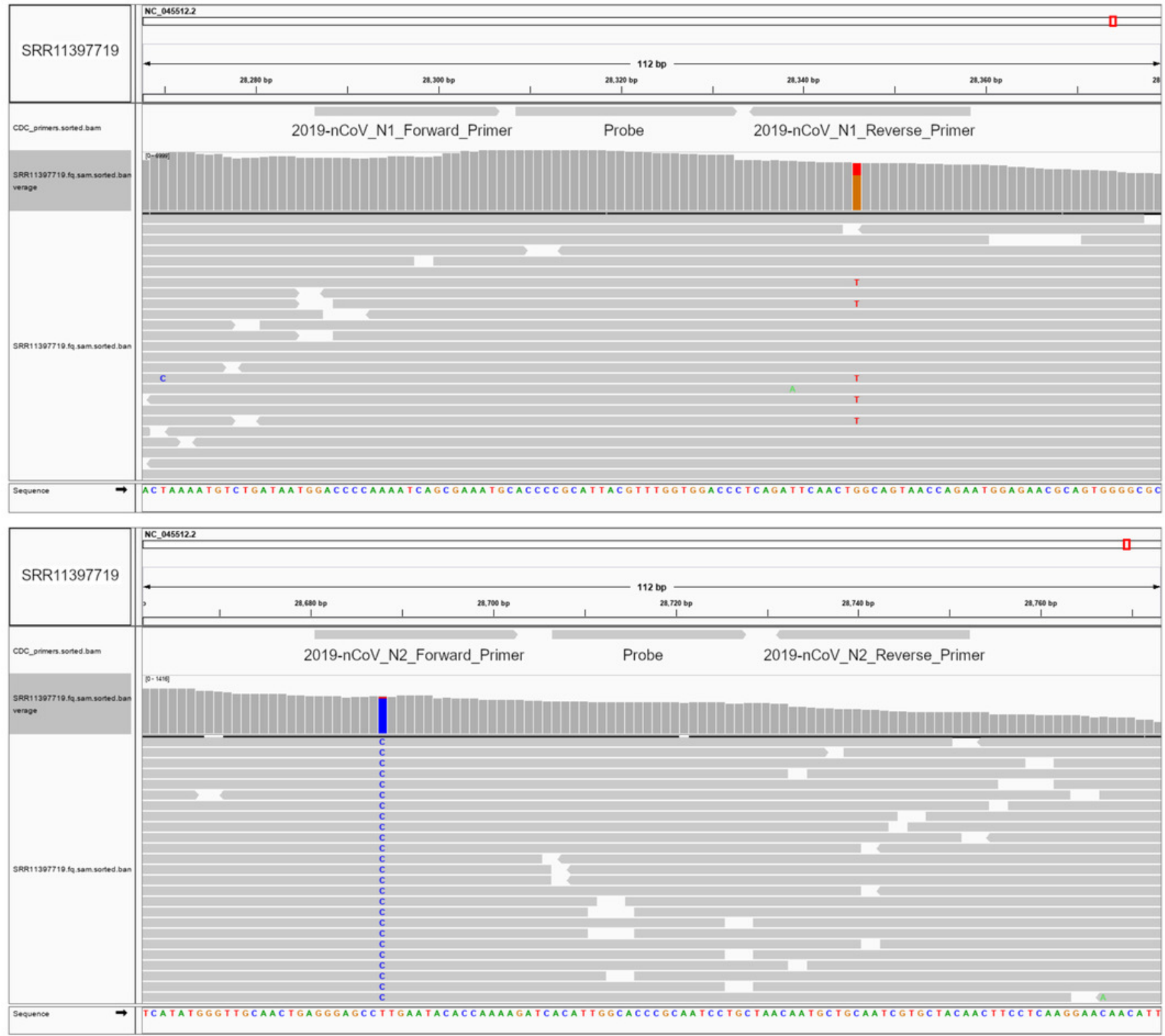


\section{Table $\mathbf{1}$ (on next page)}

Table 1: Allele frequencies of five detected variants in 1359 genbank sequences from North America

POS: position in SARS CoV 2 reference sequence NC_045512.2, REF: reference allele, ALT: mutant allele, DP4: Number of high quality ref forward, ref reverse, alt forward and alt reverse bases, AF: allele frequency. 
Table 1: Allele frequencies of five detected variants in 1359 genbank sequences from North America

\begin{tabular}{lllll}
\hline POS & REF & ALT & INFO & AF \\
8782 & $\mathrm{C}$ & $\mathrm{T}$ & $\mathrm{DP}=302 ; \mathrm{DP} 4=246,0,161,0$ & 39.55774 \\
17747 & $\mathrm{C}$ & $\mathrm{T}$ & $\mathrm{DP}=302 ; \mathrm{DP} 4=272,0,135,0$ & 33.16953 \\
17858 & $\mathrm{~A}$ & $\mathrm{G}$ & $\mathrm{DP}=302 ; \mathrm{DP} 4=273,0,134,0$ & 32.92383 \\
18060 & $\mathrm{C}$ & $\mathrm{T}$ & $\mathrm{DP}=302 ; \mathrm{DP} 4=271,0,136,0$ & 33.41523 \\
28144 & $\mathrm{~T}$ & $\mathrm{C}$ & $\mathrm{DP}=303 ; \mathrm{DP} 4=246,0,161,0$ & 39.55773 \\
\hline
\end{tabular}

POS: position in SARS-CoV-2 reference sequence NC_045512.2, REF: reference allele, ALT: mutant allele, DP4: Number of high-quality ref-forward, ref-reverse, alt-forward and alt-reverse bases, AF: allele frequency. 